\title{
Epoxy Interface Method enables Enhanced Compressive Testing of Highly Porous and Brittle Materials
}

\author{
Mehrad Mehr, Calvin Davis, Kazi Sadman, Ryan J. Hooper, Michele V. Manuel, and \\ Juan C. Nino
}

Department of Materials Science and Engineering University of Florida, Gainesville, FL 32611

mehrad@ufl.edu

(954)319-2589

\begin{abstract}
The compressive mechanical characterization of highly porous ceramics is problematic due to sensitivity to stress concentrations and localized fractures.In this paper a review of the methods used by the scientific communityis done and their typical results, advantages, and shortcomings are discussed. Here, a new methodology that can address some of the problems associated with the current measurement procedures is presented. The proposed method is applied to highly porous barium titanate foams. This procedure producesmore consistent data thatcan be analyzed and interpreted objectively. We show that properties such as the crushing or collapse stress as well as the compressive modulus cannot be extracted correctly by the conventional methods. By contrast, the proposed method collects data that is more accurate, consistent with what is expected of porous ceramics and that yields smaller measurement to measurement variation.
\end{abstract}

\section{Introduction}

Highly porous ceramics ( $>50 \%$ porosity) are used for many applications includingcatalyst structures, bio-applications, and refractories. ${ }^{1-4}$ Although porous ceramics have been investigated for decades,characterization of their mechanical 
properties remains challenging. This limitation is particularly relevant sincethe elastic and compressive behavior iscriticalforload bearing applications. For example, accurate effective modulus and compressive collapse stress are necessary for determining if certain porous ceramics are useful as bone regeneration matrices, catalytic structures, or filters. Accurate compressive testing of highly porous ceramics is difficult due to surface stress concentrations at the sample and test platen interface. ${ }^{2,5-8}$ These surface stress concentrations during compressive testing areless of a problem in ductile materials such as metallic foams. This is partially due to easier polishing and sample preparation but more importantly due to the fact that the ability of metallic foams to deform plastically prevents a cascade of localized fractures at the surface. These stress concentrations arise from the uneven load distribution due to small variations in the height of the sample at the platen interface, as seen in Figure 1. Ideally, these small variations could be eliminated with proper sample preparation. However, the brittle nature of ceramics makes polishing of highly porous samples challenging, and does not allow for deformations to ensure a uniform contact and loaddistribution at the surface. ${ }^{2}$ Therefore, as later described in detail, typical test data will be rather noisy and primarily consisting of cascaded micro-fractures andin most casesa distinct collapseor crushing stress cannot be discerned.

It is important to note that currently there aretesting standards for ordered and highly porous ceramics, e.g. honeycomb structures (ASTM C1674), but none for highly porous ceramics with randomly oriented porosity such as ceramic foams.Further, according to a news release in 2008 by ASTM International, subcommittee C28.04 was going to develop standards for porous ceramics with three-dimensional cellular ceramic foam structures. ${ }^{9}$ However, to date no new standards have been published. It is evident that a better methodologyto measure compressive response of highly porous brittle materials is necessary, such that more consistent and quantifiable data can becollected and analyzed. In this paper,two of the most widely used methods to measure compressive behavior of cellular ceramics will be reviewed; case I in which the samples are simply compressed in a universal testing machine (UTM), and case II in which compliant testing surfaces are utilized.Furthermore, a new methodology that can 
address the issues with both cases and collect more consistent data will be proposed, tested, and analyzed.

\section{Case I: Direct UTM Compression}

The most common method for characterizing the compressive behavior of porous ceramics utilizes cylindrical samples with diameters on the order of a centimeter with heights ranging from millimeters to centimeters. ${ }^{10-16}$ In some cases rectangular samples in the same size range are used ${ }^{17-25}$ and in some cases sample size is not mentioned ${ }^{26}$. Samples are usually mentioned to have been polished flat and parallel but the quality, extent, or the importance of this step is rarely emphasized. It is noteworthy that polishing can be very challenging for highly porous or weak samples due to the fact that struts tend to break instead of being polished in the grinding process. ${ }^{2}$ These samples are then placed between two platens of a mechanical testing load frame as shown inFigure 1.

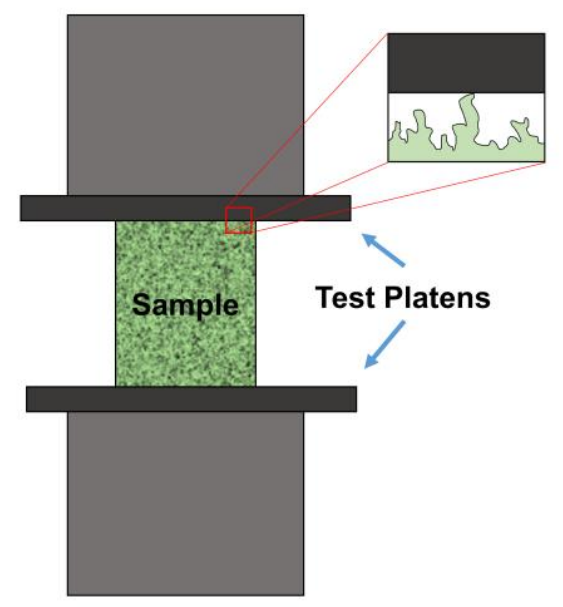

Figure 1. Schematic of case I type of compressive testing where stress concentrations may arise when samplesare placed directly between hardened steel test platens. 
The loadand displacementdatafrom the instrument are analyzed and an effective modulus(Young's modulus of the structure as a whole, not Young's modus of the struts, since stresses are defined as force divided by the area of the foam surface not accounting for porosity) and fracture/collapsestress, mostly referred to as compressive strength, is extracted from the data. It is notable that theactual stress-strain curves are often not published and only 7 of the 17 publications cited in this section present the raw data. ${ }^{11,12,14,15,17,25,26}$ Moreover, the methodology by which the effective modulus and the collapse stress areextracted is rarely discussed.Itcan be inferred in most cases that the effective modulus is defined by the ratio of stress to strain before a large drop in stress. This is commonly referred to as the collapse stress, crushing stress, fracture stress, or compressive strength. Two examples of published raw databy Zarkoob et al. ${ }^{15}$ and Gómez de Salazar et al. ${ }^{25}$ from thetype of measurement described here arereproduced after digitization in Figure 2.Zarkoob et al. investigated the effect of hydroxyapatite coatings on $78 \%$ porous disk shaped barium titanate samples. The raw mechanical data is presented by force (exerted by the samples) versus crush (displacement of testing platens) graphs. Gómez de Salazar et al. show the stress( $\sigma)$ versus displacements $(\Delta \mathrm{x})$ of rectangular $12.7 \times 12.7 \times 25.4 \mathrm{~mm}$ silicon carbide foams with 3 different ppi (pores per inch) porosity levels in which they used ASTM standard D695-96 (Standard Test Method for Compressive Properties of Rigid Plastics).
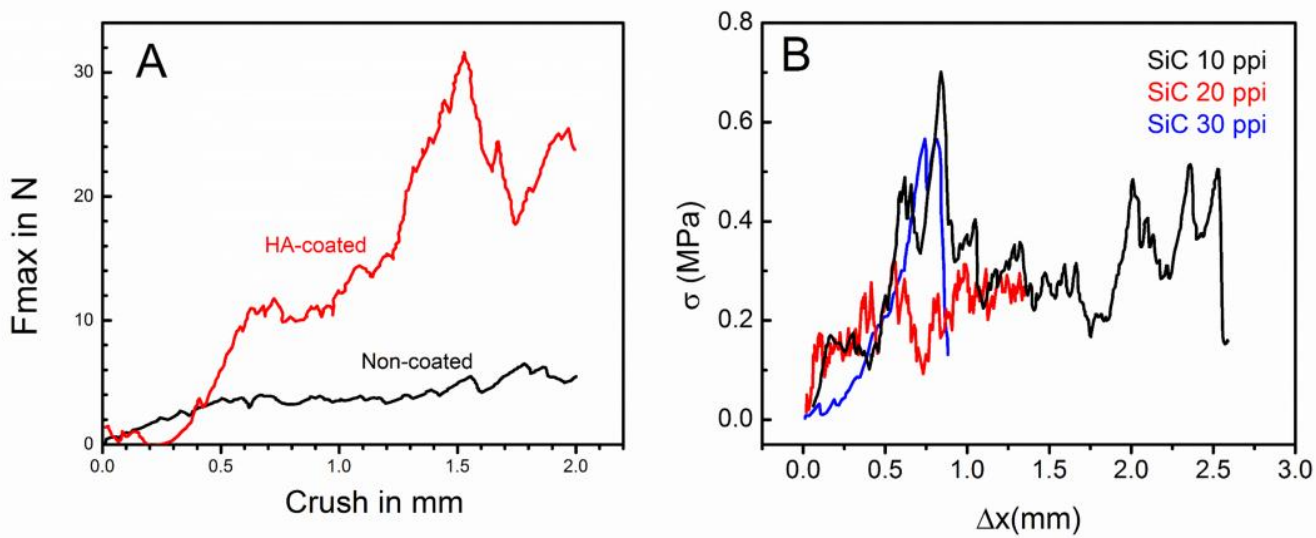

Figure 2.A) Force vs. displacement data of two different porous barium titanate samples under compression by Zarkoob et al. ${ }^{15} \mathrm{~B}$ ) SiC ceramic foam compression 
data by Gómez de Salazar et al. ${ }^{25}$

As seen in Figure 2, interpretation of stress-strain data from highly porous ceramics is challenging. The data tends to be noisy and consists of many small drops in stress whichare indicative of many small fracture events. A clear value of collapse stress is difficult to discern.For example in the case of Figure 2A, the HA coated data, it is unclear whether the sample failed at $0.7 \mathrm{~mm}$ crush or $1.5 \mathrm{~mm}$ crush $(0.4$ and $1.2 \mathrm{~mm}$ if we consider the first $3 \mathrm{~mm}$ of the test as settling even though $3 \mathrm{~mm}$ settling is very unlikely). However, it is highly unlikely that the collapse of the structure was at any of the two points $(0.7 \mathrm{~mm}$ or $1.5 \mathrm{~mm})$ since the samples used were $3 \mathrm{~mm}$ in thickness and either of the failure points would suggest that the structure was strained $23-50 \%$ (13$40 \%$ even if considering that there was $3 \mathrm{~mm}$ settling) before failure. If taken as accurate, this data would suggest that a ceramic foam structure does not fail even after being strained more than $10 \%(0.1 \mathrm{~mm} / \mathrm{mm})$. This is simply incorrect based on the brittle nature of ceramics!Further, in the case of the non-coated samples (Figure 2A, bottom curve) which were weaker, it is clear that a distinctive collapse stress characteristic of the failure of the structure was never detected and only a process of damage accumulation was observed. Consequently, the effective modulus of the structure cannot be determined accurately if small collapsesare attributed to the apparent strain in the "elastic" regime. This key aspect of inaccurate strain measurement will be revisited throughout the paper so it is important to keep it in mind.

By contrast, in Figure 2B, the strains are much more reasonable. $1 \mathrm{~mm}$ displacement on a $25 \mathrm{~mm}$ sample would be a strain of about $4 \%$ which is within reasonable expectation. However, considering that the samples vary in porosity from 10 to $30 \mathrm{ppi}$, one would expect a clearer differentiation in the compressive response.This further illustrate the challenges in determining a clear point of failure from the data collected using the direct testing method.

Another example ofstress-strain data obtained by thismethod by Jun et al. ${ }^{17}$ is shown in Figure 3 where the authors investigated the strength of highly porous ( 95\%) glass-ceramic scaffolds through direct compressive testing. 


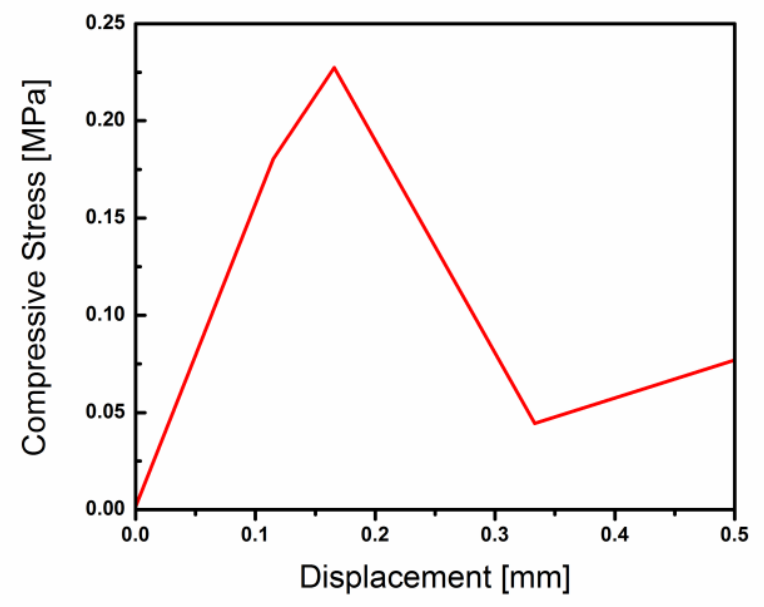

Figure 3. Example of published stress-strain data, highly porous bioactive glass-ceramics. Jun et al. ${ }^{17}$

It is important to note that although the reasons were not described in detailor discussed by the authors, the stress-strain behavior was presented as consisting of four linear segments,which is highly unlikely for any material. This suggests that either different regimes were replaced with linear fits or that only a few point were measured by the testing instrument.Regardless, the fact that this type of data is deemed acceptable for publication in one of the most important journals in ceramics further emphasizes the inconsistencies in the mechanical property measurement and analysis within the porous ceramics community, the limitations of using the conventional method of testing, and the need to develop an improved one.

\section{Case II: Compliant Interface}

The second method by which compressive behavior of highly porous ceramics is typically measured utilizes a compliant padding at both of the sample platen interfaces to achieve more uniform load distributions as shown in Figure 4. 


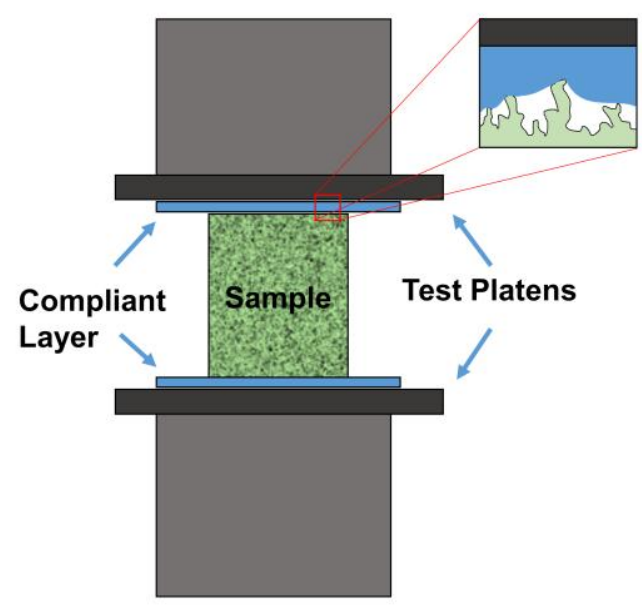

Figure 4.Schematic of case II type of compressive testing, using a compliant material between test platens and the sample.

It is worth remembering that soft padding has been used for similar reasons in testing of brittle materials for decades. ${ }^{27-29}$ More details of the use of padding and its validity in testing other materials can be found in a review by Darvel. ${ }^{30}$ One of the earliest uses of compliant padding in testing of porous ceramics was shown by Dam et al. where $3 \mathrm{~mm}$ thick neoprene was used between the samples and the platens in compression testing of open-cell alumina to in order to achieve more consistent and conclusive data. ${ }^{5}$ Others have followed this technique and it has been recommended over case I given its more consistent and reliable data. ${ }^{2}$ It is noteworthy that the compliant layer used to address the improper contact issue in testing of porous ceramics is referred to with different terminology in literature. For example Clobes and Green refer to it as bearing strips or "cushions" when using strips of manila folders to address the uniform contact issue in V-notch tests of porous alumina. ${ }^{31}$ Moreover, there are several examples of compliant interface utilized, for example, Gupta et al. used a $1 \mathrm{~mm}$ thick carbon paper ${ }^{6}$, Oliveira et al. used $2 \mathrm{~mm}$ thick PVC ${ }^{7}$, and Sandoval et al. used a combination of white and carbon papers of unspecified thickness ${ }^{8}$.Figure 5 shows examples of data collected using compliant surfaces. 

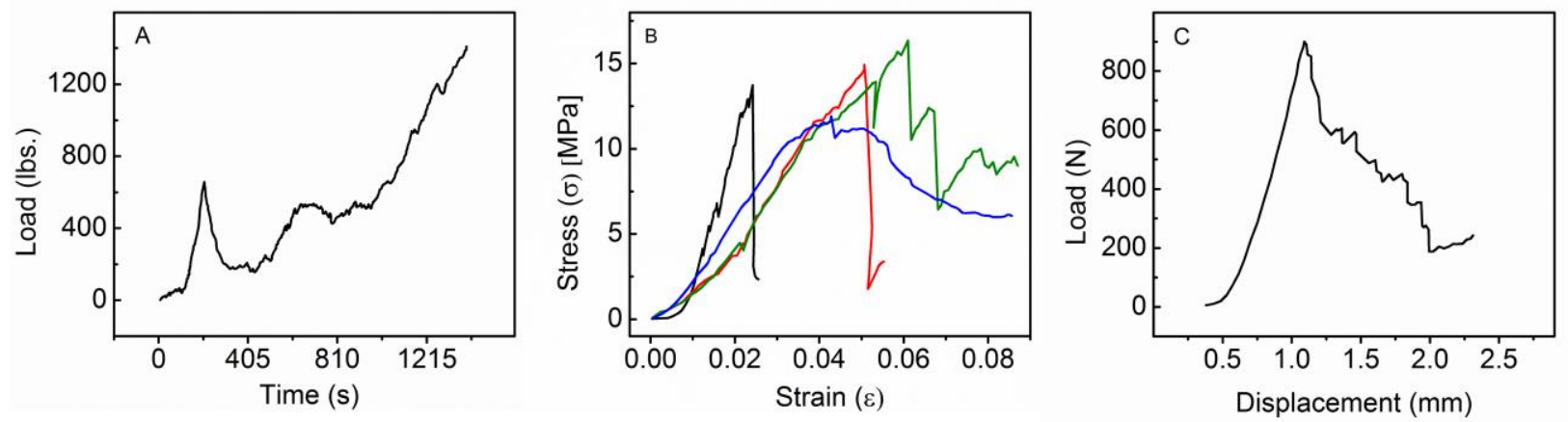

Figure 5. A) Open cell alumina by Dam et al. using neoprene. ${ }^{5} \mathrm{~B}$ ) Cordierite-based porous ceramics by Sandoval et al.using white and carbon papers. ${ }^{8}$ C) Open-cell cordierite foams by Oliveira et al. using PVC. ${ }^{7}$

As seen in Figure 5,in this case thedata reveals a fracture stress that is much more evident and can be more readily determined when compared to data from thecase I method. This is the key advantage of using compliant surfaces in testing porous brittle materials. It is noteworthy that the compliant surfaces havea minimal effect on the fracture or crushing strength of the sample from a testing standpoint since the structure is under an isostress condition. However, the compliant layers do undergo noticeable strain during testing; therefore, while stresses are measured accurately strains are not. The effective modulus calculated from this data would not be accurate unless the compressive response of the compliant layers is measured and subtracted from the data. Neoprene as an example of such compliant material can have strains up to $90 \%$ under a stress of about 1-2 MPa and about $50 \%$ strain under a stress of about $0.2 \mathrm{MPa}$ as shown in Figure 6by Bardy et al. ${ }^{32}$ 


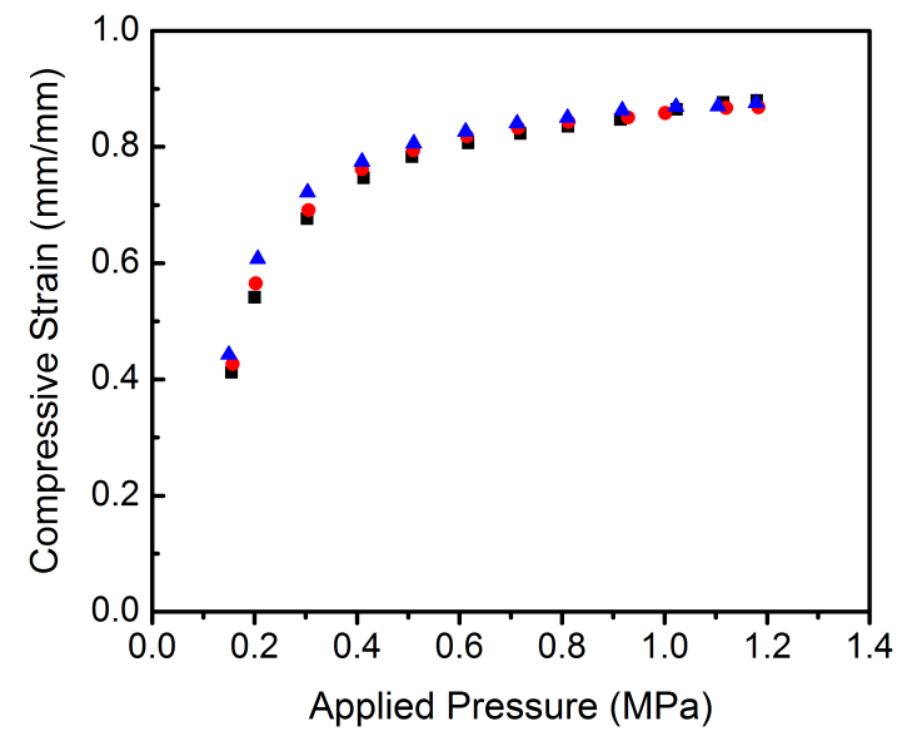

Figure 6.Uniaxial compression behavior of neoprene. Data by Bardy et al. ${ }^{32}$

As a result of this, the data in Figure 5aand Figure $5 \mathrm{c}$ were not presentedas the stressstrain response of the material by the authors. In these two examples the compliant layers are thick compared to the samples andthus can significantly contribute to the displacement. In the work by Sandoval et al. ${ }^{8}$ in Figure $5 \mathrm{~b}$ the compliant layers were thin enough in comparison to the samples for the contributed error to be considered negligible by the authors and the data to be presented as a stress-straincurve.

There are two main scenarios for the use of compliant layers. First, a sample is being tested in a stress region where the compressive strain of the compliant layer has not plateaued yet (e.g. $\sim 1 \mathrm{MPa}$ for neoprene, ignoring any strain rate dependence), in which case large strains from the layer will contribute to the total strain and the error will be significant.For example, in a samplewith a height of $1 \mathrm{~cm}$ with $1 \mathrm{~mm}$ of neoprene on each side ( $2 \mathrm{~mm}$ total), the strain from the porous ceramic can usually be about $<5 \%$ while the compliant layer $(50-75 \%$ compression, $1 \mathrm{~mm}-1.5 \mathrm{~mm})$ will contribute another 10 to $15 \%$.It is possible to extract the added strain from the data afterwards but the practice could raise its own difficulties and concerns (an example of these concerns is in terms of contact mechanics, strains resulting from penetration of an array of struts varying in height, as depicted in figure 4 , is much different than the compressive response of the 
complaint material tested against flat platens). The second scenario occurs if the data collection begins after the majority of the compliant layer deformation has occurred. In that case, the Poisson's ratio of the compliant layer material and its outward lateral strainwill induce shear forces on the faces of the sample and may cause the structure to fail under an axial cleavage mode as well as barreling due to friction effectsrather than internal cracking. ${ }^{33}$ For highly porous ceramics this mode of failure would be likely. Some of these issues could be addressed by using less compliant materials but then the original problem of surface stress concentrations will rise again. Therefore, a compromise is necessary, and proper compliant material selection must be made based on a thorough understanding of the compressive behavior of the layer.In conclusion, while this technique is currently the best in studying compressive behavior of highly porous brittle materials it still has shortcomings and thus an improved methodology is proposed.

\section{Summary of current testing methods}

The majority of compressive testing on highly porous ceramic materials is done by direct placement of the sample in a universal testing machine. The porous nature of the surface as well as the brittleness of the material prevents proper sample platen contact and as a result a series offailures at the improper contact locationsare recorded by the testing instrument. Accurate load bearing properties of the material such as the effective modulus $\left(E^{*}\right)$ and compressive strength $\left(\sigma^{*}\right)$ as described in the theoretical model of Gibson and Ashby ${ }^{34}$ cannot be extracted. In most cases the first major failure of the structure is identified as the collapse of the structure and the prior regime is called the elastic regime. However, this conclusion is false considering the high strains (10$20 \%$ ) at which they occur. Many in the community have realized this problem and have addressed the issue by using a compliant layer at the sample-platen interface. Nonetheless, this alternative solution doesnot resolve the problem. While, the strain values at which the failureoccurs are much more reasonable, the compliant layers used add significant strain (with respect to few percent strains expected from the samples), and thus theobtained measurements are only accurate for extracting collapse or crushing stress. The goal of this paper is to propose a new method which addresses 
these issues and therefore vastly improves on the methodology currently used and accepted by the community.

\section{Proposed Method: Conforming but Stifflnterface}

In a previous study,compressive mechanical properties of barium titanate foams were investigated. ${ }^{35}$ In that work a confinement cell was used for better loading of the samples and prevention of buckling.Confinements and the methods used had been shown to be useful in other studies on polymeric foams. ${ }^{36,37}$ Strain was extracted directly from the sample using a video recording of the grid lines on the sample rather than the testing instrument displacement.The displacement of the grid lines were then used to calculate straindirectly from the sample.While that technique can be useful in certain cases and can produce data for comparative and qualitative analysis, it cannot accurately measure the collapse strength and the effective modulus of the structure for ceramic foams. This is because the localized surface fractures (problem that is being addressed in this paper) prevents a clear identification of the initial collapse of the structure. Even if the initial collapse does clearly prevail, the strain inceramic foams, which is on the order of a few percent, will be hard to resolve with grid lines. However, the confinement itself drastically simplifies handling, sample alignment and orthogonal loading during testing which is very important for collecting reliable data and thus it is used here, although it is not indispensable for the here proposed methodology.

The methodology presented here takes advantage of the confinement method but most importantly it improves upon methods to uniformly distribute stress at the platen-sample interface to collect more accurate data. In order to make uniform contact with the sample it is clear that the boundary layer material must have a high compliancesuch that it can more adequatelyconform to the contours of the sample surface. Ideally, the material would be a viscous liquid with a high enough viscosity such that it does not flow through the pore windows beyond the surface pores of the sample. However, to avoid problems described in case II the layer must be significantly stiff to prevent extensive deformation during testing. Therefore it isproposed that a highmodulus(relative to the sample, for example two orders of magnitude higher to minimize strain errors, details of the contribution to error is described in the results 
section) epoxy can be applied to both surfaces of the sample as a viscous liquid (before curing) to ensure surface conformation, and after the epoxy is curedsamples can be tested taking advantage of the newly formed rigid interface as shown inFigure 7. In essence, the layer allows uniform contact with the surface while itself not deforming during testing. In this scenario the (effectively) infinitely rigid layer can be considered a part of the testing instrument and not participating in measurements in the same manner as the platens of the universal testing machine itself.

\section{Experimental Methods}

For the highly porous ceramic samples needed, barium titanate $\left(\mathrm{BaTiO}_{3}\right)$ foams were chosen because of their ease of processing, sample consistency, and previous line of work. $\mathrm{BaTiO}_{3}$ foams were made through a direct foaming method utilizing a polyurethane system. The details of the synthesis of the $\mathrm{BaTiO}_{3}$ foams can be found elsewhere. ${ }^{38}$ Samples were cut into cylinders with a diameter of $12.7 \mathrm{~mm}$ using a steel coring blade. Their surfaces were ground flat and parallelwith a 320 grit and subsequently a 600 grit silicon carbide paper with minimal force in order to avoid fracture of surface struts (as much as possible) and produce most suitable samples for characterization using the conventional case I method. The height of all samples(ranging from $7-14 \mathrm{~mm}$ ) were recorded and used for strain calculations in the 

system, hardener 20-3578 and resin 20-3576 (1:2 volumetric ratio), and were left overnight to dry. Thesame procedure was followed forboth surfaces.As shown in our previous work,compressive testing of the samples was done using an acrylic confinement cell to avoid buckling ${ }^{36}$ and provide sample alignment for orthogonal loading.Compression testing was carried out on an Instron 5582 load frame. Each test was run at a constant crosshead displacement of $1.27 \mathrm{~mm} / \mathrm{min}$. Sample displacement measurements were obtained using a calibrated non-contact laser extensometer from Electronic Instrument Research (Model LE-05, resolution of $0.001 \mathrm{~mm}$ ) with references located on the confinement cell to avoid measuring any instruments strains. The force was applied by a spherically-seated compression platen, which compensates for nonparallel features on the sample fixtureAll samples were foamed, sintered, and tested in the same orientation to account for anisotropy and were randomly assigned to each experimental group. All samples were foamed at the same time to minimize variations since factors such as humidity can affect porosity (relative density) of the foams. In a separate set of experiments it was confirmed that the relative density of the foams synthesized using the procedure mentioned here were $0.11 \pm 0.01$ and all samples are expected to be in this range. Seven samples were tested for both epoxy and non-epoxy measurements.

\section{Results and Discussion}

A scanning electron micrograph of the sample surface is shown in Figure 8. The surface microstructure demonstrates the irregularities of the surface and that few struts may come in contact with a flat surface. The applied layer of epoxy on $\mathrm{aBaTiO}_{3}$ foam sample is shown in Figure 9. Epoxy caps with thickness of $0.8-1.2 \mathrm{~mm}$ at the center of the cap were able to be reproduced for every sample surface by applying a few drops of the very viscus epoxy mixture to the surface. The high viscosity of the epoxy mixture insured that it did not flow beyond the surface cells of the foams due to gravity or capillary effects. This was confirmed by experiments were the epoxy caps were separated from the foam surfaces which revealed that the epoxy only coated the surface. 


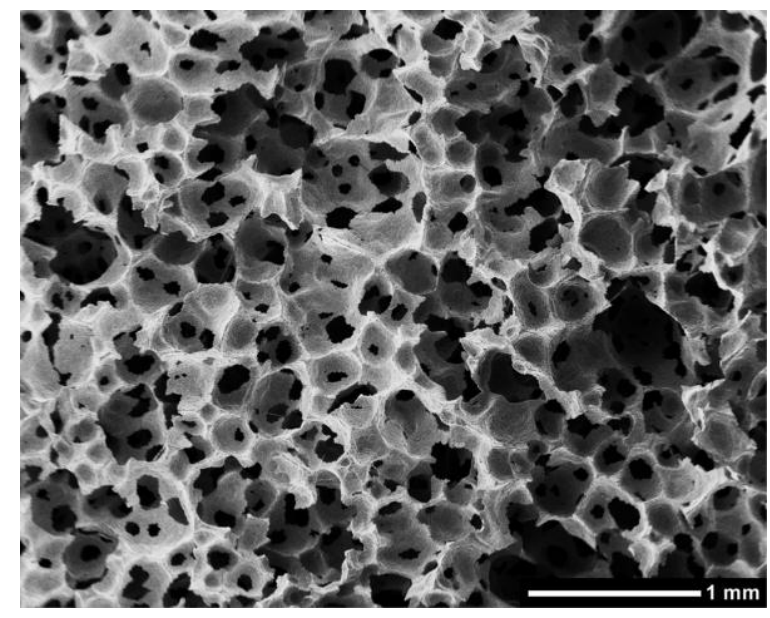

Figure 8. Scanning electron

micrograph of the barium titanate foam sample surface.

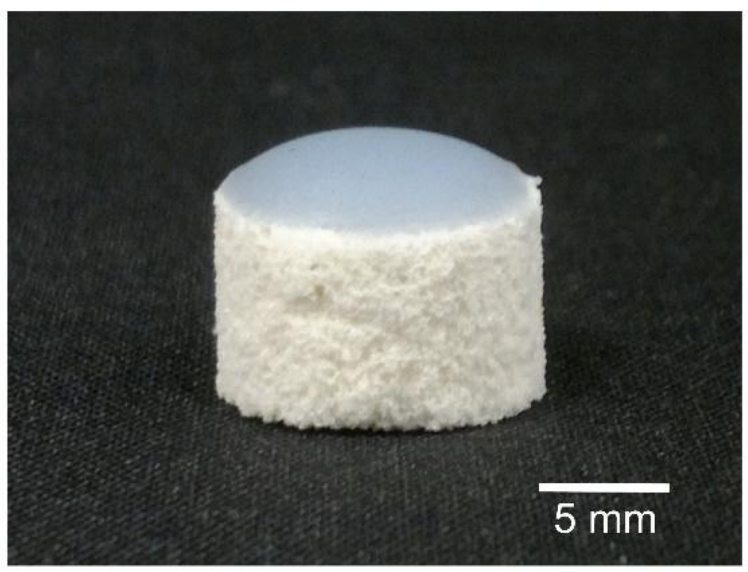

Figure 9. Barium titanate foam sample with dried epoxy cap on testing surface.

Load and displacement data collected through both the conventional (case I) method and the proposed method with epoxy caps are converted to stress and strain (calculated by dividing the displacement measured by the laser extensometer by the original height of the samples without epoxy caps) and are shown in Figure 10. 

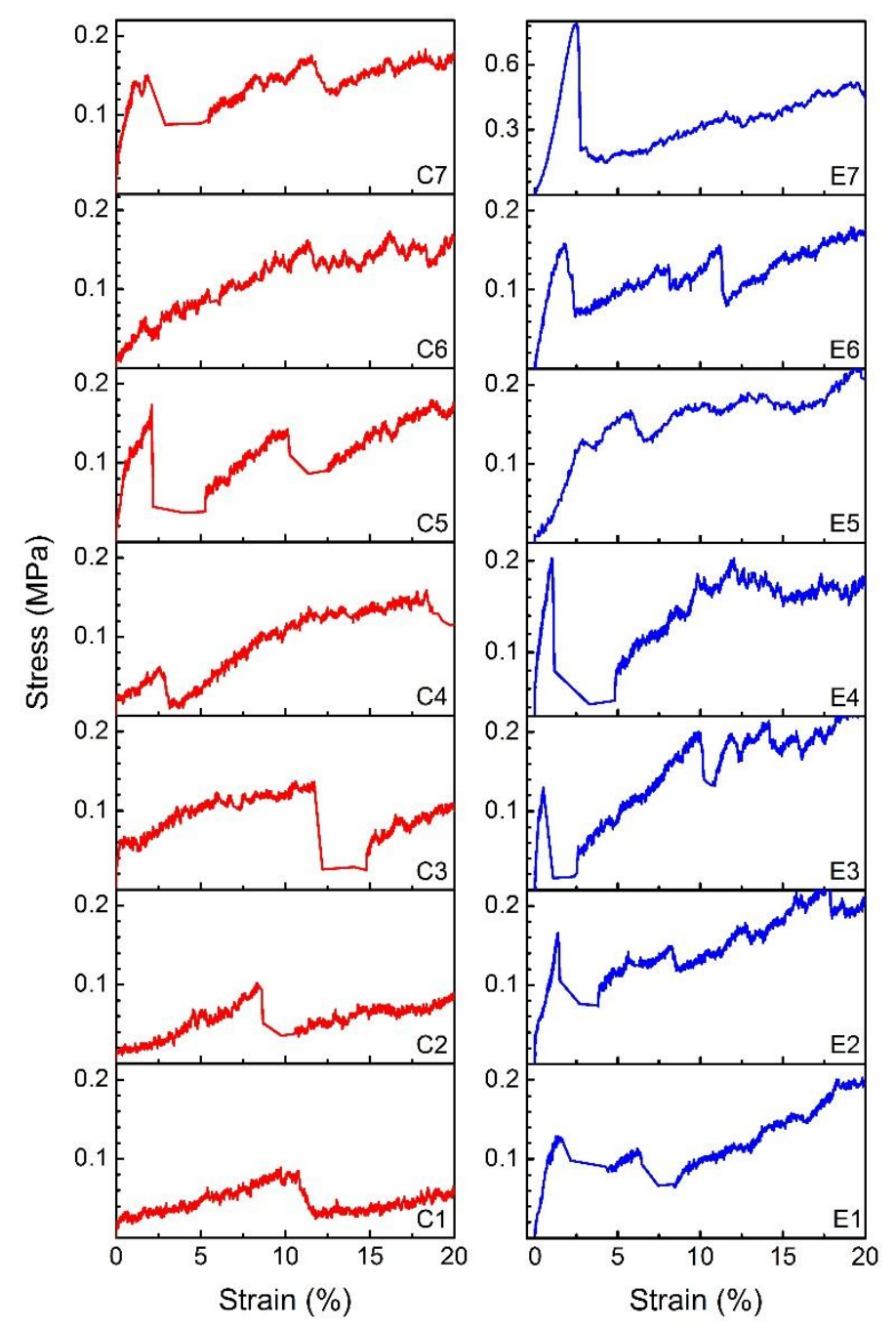

Figure 10.Stress-strain data collected through conventional (red) and proposed method (blue). All data on same scale except E7.

The samples tested using epoxy (E1-E7)are more consistent interms of characteristic features than those measured conventionally $(\mathrm{C} 1-\mathrm{C} 7)$. Nearly all samples have the following characteristics which are consistent with the explanation of each region given by Dam et al..$^{5}$ Compressive response begins with a rapid increase in stress which is called the elastic region. In this region the overall structure has not collapsed,however, small variations in stress in this region may be present which suggest failure of internal struts of the foam which would decrease the overall force exerted by the sample. The 

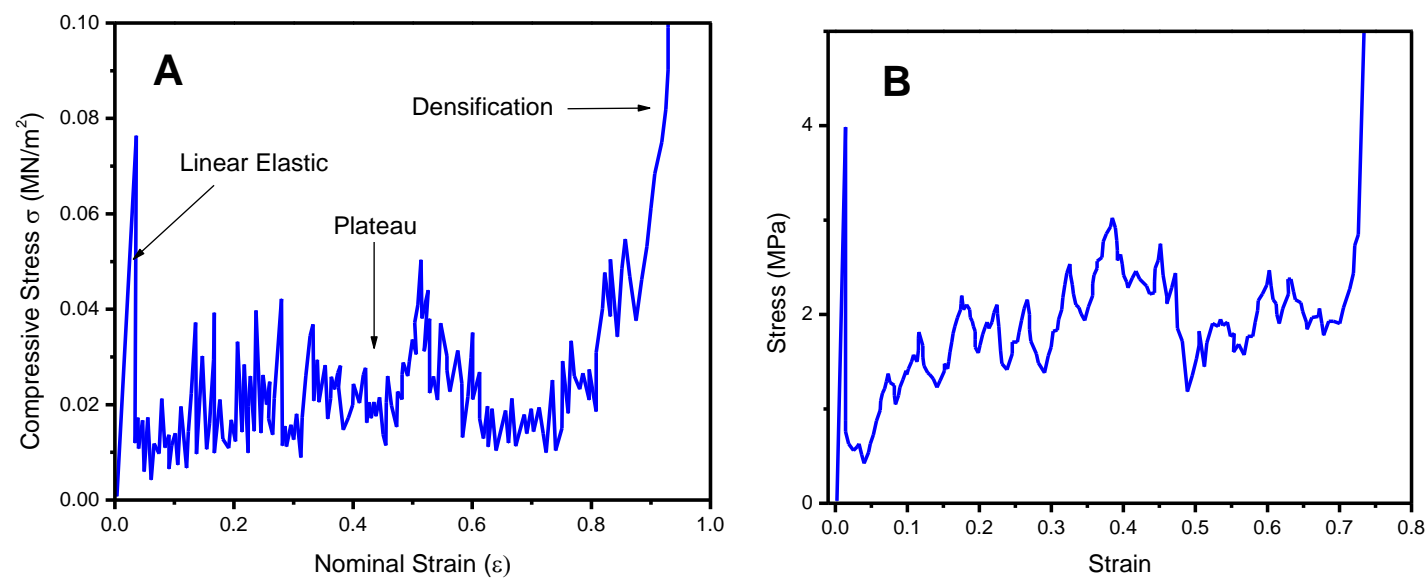

Figure 11.A) Stress-strain response of (mullite) presented by Gibson and Ashby as nominal response of porous brittle material ${ }^{34}$ B) Stress-strain response of highly porous ceramics measured by X-ray computed tomography by Caty et al. ${ }^{39}$ 
It is notable that the data collected through the conventional methods here has more apparent collapse points in comparison to other examples from literature(see Figure 2, for example). This can be contributed by the quality of the groundsurfaces used here that help ensure the best results through the conventional method.

To contrast the experimental results following both methods, Table 1 shows the collapse stress, collapse strain, and the effective modulus (slope of a line fitted through the stress-strain data from no strain to the point of collapse) of the $\mathrm{BaTiO}_{3}$ foams.

Table 1. Collapse stress, collapse strain, and effective modulus of samples measured through the conventional and the proposed method.

\begin{tabular}{|c|c|c|c|c|c|c|}
\hline & \multicolumn{3}{|c|}{ Conventional Method(C) } & \multicolumn{3}{c|}{ Proposed Method(E) } \\
\hline $\begin{array}{c}\text { Sample } \\
\text { number }\end{array}$ & $\begin{array}{c}\text { Collapse } \\
\text { Stress (MPa) }\end{array}$ & $\begin{array}{c}\text { Collapse } \\
\text { Strain } \\
(\mathrm{mm} / \mathrm{mm})\end{array}$ & $\begin{array}{c}\text { Effective } \\
\text { Modulus (MPa) }\end{array}$ & $\begin{array}{c}\text { Collapse } \\
\text { Stress (MPa) }\end{array}$ & $\begin{array}{c}\text { Collapse } \\
\text { Strain } \\
(\mathrm{mm} / \mathrm{mm} \text { ) }\end{array}$ & $\begin{array}{c}\text { Effective } \\
\text { Modulus (MPa) }\end{array}$ \\
\hline 1 & 0.078 & 0.11 & $0.59 \pm 0.006$ & 0.13 & 0.014 & $7.9 \pm 0.2$ \\
\hline 2 & 0.097 & 0.084 & $1.0 \pm 0.009$ & 0.17 & 0.014 & $10 \pm 0.01$ \\
\hline 3 & 0.13 & 0.12 & $0.67 \pm 0.009$ & 0.13 & 0.0049 & $21 \pm 0.5$ \\
\hline 4 & 0.057 & 0.026 & $1.6 \pm 0.04$ & 0.2 & 0.011 & $13 \pm 0.2$ \\
\hline 5 & 0.17 & 0.021 & $5.6 \pm 0.1$ & 0.13 & 0.029 & $4.4 \pm 0.1$ \\
\hline 6 & $\mathrm{NA}$ & $\mathrm{NA}$ & $\mathrm{NA}$ & 0.16 & 0.018 & $9.0 \pm 0.2$ \\
\hline 7 & 0.14 & 0.012 & $6.4 \pm 0.2$ & $\mathrm{NA}$ & $\mathrm{NA}$ & $\mathrm{NA}$ \\
\hline Average & 0.110 & 0.061 & 2.60 & 0.150 & 0.015 & 11.0 \\
\hline STDV & 0.043 & 0.047 & 2.40 & 0.029 & 0.0080 & 5.3 \\
\hline Coeff. of variation & 0.38 & 0.77 & 0.920 & 0.19 & 0.54 & 0.48 \\
\hline$\%$ decrease in C.V. & & & & 50 & 29 & 47 \\
\hline
\end{tabular}

$\%$ decrease in C.V.

50

Sample C6 had no clearcollapse stressin the strain range investigated and was not included in the results but presented here for completion. Inconclusive test resultssuch as C6 is suspected to be common for weak and highly porous ceramics tested through the conventional method. SampleE7 was confirmed to be an outlier by both the Dixon's $(P<0.002)$ and Grubbs's $(P<0.001)$ test for single outliers and wasnot included in the results. E7 was almost 5 times stronger than other samples. This is suspected to be due to processing and is not considered to be a fault of the measurement, but rather a reminder of the challenge of attaining sample uniformity in typical foamed ceramics. For example, the direct foaming method used in the present samples is highly sensitive to 
water content during the foaming process, and therefore, an experimental error with the micro pipette (i.e. incomplete dispensing) leading to slightly less water content can result in lower porosity, higher relative density, and much stronger foams. Using the proposed method, the coefficient of variation of the collapse stress, collapse strain, and effective modulus decreasedby 50,29 , and $47 \%$, respectively. The net result is that, as observed in literature, the conventional methodsyields data that are not only far from the nominal response, but also are more inconsistent in comparison to the epoxy method.

As described before, another salient characteristicthat highlights the inconsistency in the conventional method is the high level of collapse strains in samples. For example in C1 ,C2, and C3 collapse strain is nearly 10\%, much higher than expected for a brittle ceramic. The high strain is due to improper contact and a cascade of small fractures (damage accumulation as sometimes called) at the surface due to stress concentrations. By contrast, the strain values rangingfrom 1 to $3 \%$ measured through the proposed method and sample $\mathrm{C} 5$ and $\mathrm{C} 7$ are more fitting for a brittle ceramic.

Strain Error from the Epoxy Layer. As mentioned in the Case II, the compliant interface utilized resulted in a large error in the measured strain during testing. Therefore, in order to calculate the error resulting from the epoxy layer, the compressive stress-strain data for an epoxy disk was measured (not shown).The compressive modulus of the epoxy used was 1.4 GPa, and the strain of the epoxy in the stress regime of our test ( $0-0.5 \mathrm{MPa}$ to be conservative) was only $0.035 \%$. That means that the displacement due to a $2 \mathrm{~mm}$ ( $1 \mathrm{~mm}$ on each side) epoxy layer during testing would only be on the order of $10^{-4} \mathrm{~mm}$. An added displacement of this magnitude contributesto a strain error on the order of $10^{-5}$ for samples with a height of $\sim 1 \mathrm{~cm}$. It is clear that the introduced error in the strain measurements is negligible.

However, to be more precise, it is important to notethat the surface tension of the uncured epoxy creates a spherical surface. The cured epoxy can be assumed infinitely rigid in comparison to the test specimen. Thus, the slight spherical nature of the epoxy cap is not expected to add significant error to the measurement and transfers the load uniformly to the sample. The epoxy caps could be ground to have a flat surface, however, the spherical nature of the cap may in fact be advantageous by compensating 
for slightly unparalleled sample surfaces. This is for example exploited in spherically seated test platens and spherical load transfer parts in ASTM standards E9-09 and C165-07 for compression testing.Nonetheless, it is clear that when using this testing arrangement one must be mindful of the contact mechanics and deformations at the point of contact between the epoxy and test instrument.

Moreover, although the epoxy layer may be considered infinitely rigid with respect to sample stresses, stress concentration at the point of contact may cause deformations and contribute strain errors during testing. These deformations can be calculated using Hertzian contact stress analysis. The radius of the contact surface (a) between two elastic spheres is given by equation $1:{ }^{40}$

$$
a=\sqrt[3]{\frac{3 F\left[\frac{1-v_{1}^{2}}{E_{1}}+\frac{1-v_{2}^{2}}{E_{2}}\right]}{4\left(\frac{1}{R_{1}}+\frac{1}{R_{2}}\right)}}
$$

where $F$ is the load, $R_{1}$ and $R_{2}$ are the radii, $E_{1}$ and $E_{2}$ are the elastic moduli, and $v_{1}$ and $v_{2}$ are the Poisson's ratios of sphere 1 and 2 respectively. For the case of the epoxy cap contacting the testing instrument platen, $R_{2}$ (radius of platen) and $E_{2}$ (modulus of the platen) are infinite since the hardened steel platen of test frame is flat and is infinitely rigid with respect to the epoxy cap. $R_{1}$, the radius of curvature of the epoxy cap, can be estimated using the equation 2 for the length of a circle secant:

$$
R=\frac{h}{2}+\frac{c^{2}}{8 h}
$$

where $R$ is the radius, $c$ is the secant length, and $h$ is the height of the circle segment. In the case of the epoxy caps used here, the height of the segment is $\approx 1 \mathrm{~mm}$, and $c$ is the diameter of the samples $\approx 12.7$ mmapproximating the radius of curvature to be about $20 \mathrm{~mm}$. Using this value in equation 1 for $R_{1}$, compressive modulus of $1.4 \mathrm{GPa}$ and a Poisson's ratio of 0.3 the radius of contact surface between the epoxy and the testing surface is $\approx 0.6 \mathrm{~mm}$. Using equation 2 the height of the deformed segment of the epoxy cap can be calculated to $\approx 0.009 \mathrm{~mm}$. Therefore the displacement due to the top and bottom epoxy caps would be $\approx 0.018 \mathrm{~mm}$ contributing a strain error of $\approx 0.1 \%$ on $1 \mathrm{~cm}$ 
samples. This error is noticeably larger than previous assumption of a flat cap but still remains negligible in comparison of the strains measured.

Finally the strain error contributed from the acrylic confinement cell used in this study must be analyzed. Loads were applied by thecylindrical rod of the confinement cell. The length of the rod from the point of reference where strain measurements were made to the point where it made contact with the sample was $8 \mathrm{~cm}$. The elastic modulus of acrylic is about 2-3 GPa. ${ }^{41}$ This yields a displacement error of about 5-8 $\times$ $10^{-4} \mathrm{~cm}$ under $\sim 0.2 \mathrm{MPa}$ test stresses and a negligible strain error on the order of $10^{-4} \mathrm{or}$ $10^{-2} \%$.

\section{Conclusion}

The mechanical properties of porous ceramics are difficult to obtain and interpret from compression tests. Specifically, the stress concentrations at the sample and test platen interface have made compressive testing of porous ceramics problematic.Nonetheless, these tests are widely used by the porous ceramics community. Typical compressive testing of highly porous ceramics yields noisy data with high variability among samples. More importantly, the interpretation of stress-strain data is subjective in nature and properties such as the effective modulus or the collapse stress are extracted widely differently across literature. An improvement ofthe compression testingmethodology is needed for the collection of more consistent data and in returnattain better models describing the mechanical behavior of these materials. The application and use of epoxy layerswith high compressive modulus on each surface of the porous ceramic samplesis shown here to resolve the problem without introducing any significant error to the data.In this study, the data obtained using epoxy capsarein agreement with expected response of this class of materials. Data ismore consistent, less noisy, and can be analyzed objectively in comparison to the conventional types of compression testing. Although in this study a confinement cell was utilized, it is expected that the epoxy cap has very similar effect on data collection without confinement. The properties extracted from the data had a coefficient of variation about $50 \%$ less than data collected through conventional testing. Strain errors due to epoxy 
caps are expected to be at most $0.1 \%$. Future studies on other systems with samples of varying sizes are necessary to further validate this technique.

\section{Acknowledgments}

We gratefully acknowledge Professor Jack Mecholsky for his helpful discussions regarding this work. We also thank Bruno Santos for his helpin the manuscriptpreparation.Mehrad Mehr is supported under a Department of Energy Nuclear Energy University Programs Graduate Fellowship.

\section{References}

1 A. Julbe, D. Farrusseng, and C. Guizard, "Porous ceramic membranes for catalytic reactors - overview and new ideas," J. Membr. Sci., 181 [1] 3-20 (2001).

2 M. Scheffler and P. Colombo, Cellular ceramics: structure, manufacturing, properties and applications. John Wiley \& Sons, 2006.

3 E. Litovsky, M. Shapiro, and A. Shavit, "Gas pressure and temperature dependences of thermal conductivity of porous ceramic materials .2 . Refractories and ceramics with porosity exceeding 30\%," J. Am. Ceram. Soc., 79 [5] 1366-1376 (1996).

4 E. Kon, A. Muraglia, A. Corsi, P. Bianco, M. Marcacci, I. Martin, A. Boyde, I. Ruspantini, et al., "Autologous bone marrow stromal cells loaded onto porous hydroxyapatite ceramic accelerate bone repair in critical-size defects of sheep long bones," J. Biomed. Mater. Res., 49 [3] 328-337 (2000).

5 C.-Q. Dam, R. Brezny, and D.J. Green, "Compressive behavior and deformation-mode map of an open cell alumina," Journal of Materials Research, 5 [1] 163-71 (1990).

$6 \quad$ R.K. Gupta, E.Y. Kim, H.S. Noh, and C.M. Whang, "Mechanical, electrical and microstructural properties of La0.6Sr0.4Co0.2Fe0.8O3 perovskite-based ceramic foams," J. Phys. D: Appl. Phys., 41 [3] 032003 (2008). 
7 F.A. Oliveira, S. Dias, M.F. Vaz, and J.C. Fernandes, "Behaviour of open-cell cordierite foams under compression," Journal of the European Ceramic Society, 26 [1] 179-186 (2006).

8 M.L. Sandoval, M.A. Camerucci, and A.G.T. Martinez, "High-temperature mechanical behavior of cordierite-based porous ceramics prepared by modified cassava starch thermogelation," J Mater Sci, 47 [23] 8013-8021 (2012).

$9 \quad$ ASTM International News Releases, Flexural Testing of Honeycomb Ceramics Covered in New ASTM International Standard, Release \#8055, (2008).

10 G. Wei and P.X. Ma, "Structure and properties of nano-hydroxyapatite/polymer composite scaffolds for bone tissue engineering," Biomaterials, 25 [19] 4749-4757 (2004).

11 N. Altinkok, A. Demir, I. Ozsert, and F. Findik, "Compressive Behavior of Al2O3-SiC Ceramic Composite Foams Fabricated by Decomposition of Aluminum Sulfate Aqueous Solution," Journal of Composite Materials, 41 [11] 1361-1373 (2007).

12 S. Meille, M. Lombardi, J. Chevalier, and L. Montanaro, "Mechanical properties of porous ceramics in compression: On the transition between elastic, brittle, and cellular behavior," Journal of the European Ceramic Society, 32 [15] 3959-3967 (2012).

13 J.C. Le Huec, T. Schaeverbeke, D. Clement, J. Faber, and A. Le Rebeller, "Influence of porosity on the mechanical resistance of hydroxyapatite ceramics under compressive stress," Biomaterials, 16 [2] 113-118 (1995).

14 Y. Ota, T. Kasuga, and Y. Abe, "Preparation and Compressive Strength Behavior of Porous Ceramics with $\beta$-Ca (PO3) 2 Fiber Skeletons," Journal of the American Ceramic Society, 80 [1] 225-231 (1997).

15 H. Zarkoob, S. Ziaei-Rad, M. Fathi, and H. Dadkhah, "An investigation on mechanical behavior of barium titanate foam with hydroxyapatite coating," Ceramics International, 38 [4] 3445-3451 (2012). 
16 Highly Porous YSZ Ceramics After Silica Aerogels Impregnation,” J. Am. Ceram. Soc., 96 [10] 3223-3227 (2013).

17 I.-K. Jun, Y.-H. Koh, and H.-E. Kim, "Fabrication of a highly porous bioactive glass-ceramic scaffold with a high surface area and strength," Journal of the American Ceramic Society, 89 [1] 391-394 (2006).

18 D.-M. Liu, "Influence of porosity and pore size on the compressive strength of porous hydroxyapatite ceramic," Ceramics International, 23 [2] 135-139 (1997).

19 Z. Lu, Q. Liu, H. Han, and D. Zhang, “Experiment and modeling on the compressive behaviors for porous silicon nitride ceramics," Materials Science and Engineering: A, 559 201-209 (2013).

20 Oxycarbide Ceramic Foams," Journal of the American Ceramic Society, 84 [10] 2245-2251 (2001).

21 J. Du, X. Zhang, C. Hong, and W. Han, "Microstructure and mechanical properties of ZrB2$\mathrm{SiC}$ porous ceramic by camphene-based freeze casting," Ceramics International, 39 [2] 953-957 (2013).

22 I. Yakub, J. Du, and W.O. Soboyejo, "Mechanical properties, modeling and design of porous clay ceramics," Materials Science and Engineering: A, 558 21-29 (2012).

23 R. Ahmad, J.-H. Ha, and I.-H. Song, "Effect of valeric acid on the agglomeration of zirconia particles and effects of the sintering temperature on the strut wall thickness of particlestabilized foam," Journal of the European Ceramic Society, 34 [5] 1303-1310 (2014). Y. Yang, S. Shimai, Y. Sun, M. Dong, H. Kamiya, and S. Wang, "Fabrication of porous Al2O3 ceramics by rapid gelation and mechanical foaming," Journal of Materials Research, 28 [15] 2012-2016 (2013). 

fracture toughness test for porous alumina," Journal of Materials Science, 37 [12] 24272434 (2002). Geology, 4 [3] 179-285 (1970). press, 1997. 
35 L. Wucherer, J.C. Nino, and G. Subhash, "Mechanical properties of BaTiO $<$ sub $>3</$ sub $>$ open-porosity foams," Journal of the European Ceramic Society, 29 [10] 1987-1993 (2009).

36 Q. Liu and G. Subhash, "A phenomenological constitutive model for foams under large deformations," Polym Eng Sci, 44 [3] 463-473 (2004).

37 T.R. Walter, A.W. Richards, and G. Subhash, "A Unified Phenomenological Model for Tensile and Compressive Response of Polymeric Foams," Journal of Engineering Materials and Technology, 131 [1] 011009 (2009).

38 L. Wucherer, J.C. Nino, F. Basoli, and E. Traversa, "Synthesis and Characterization of BaTiO3-Based Foams with a Controlled Microstructure," International Journal of Applied Ceramic Technology, 6 [6] 651-660 (2009).

39 O. Caty, F. Gaubert, G. Hauss, and G. Chollon, "Characterisation of mechanical properties of cellular ceramic materials using X-Ray computed tomography”; pp. 39-46 in Optical Measurements, Modeling, and Metrology, Volume 5. Edited by T. Proulx. Springer New York, 2011.

40 S. Timoshenko, Theory of Elasticity. McGraw-Hill, 1934.

41 B. Fry, Working with Acrylic. Society of Manufacturing Engineers, 1999. 